\title{
Molecular emulsions: from charge order to domain order
}

\author{
Aurélien Perera
}

October 3, 2018

Laboratoire de Physique Théorique de la Matière Condensée (UMR CNRS 7600), Université Pierre et Marie Curie, 4 Place Jussieu, F75252, Paris cedex 05, France.

\begin{abstract}
Aqueous mixtures of small molecules, such as lower n-alkanols for example, are known to be micro-segregated, with domains in the nano-meter range. One consequence of micro-segregated domains would be the existence of long range domain-domain oscillatory correlations in the various atom- atom pair correlation functions, and subsequent pre-peaks in the corresponding atom atom structure factors, in the q-vector range corresponding to nano-sized domains. However, no such pre-peak have ever been observed in the large corpus of radiation scattering data published so far. Here, through large scale simulations of aqueous-1propanol mixtures, I report that the domain pre-peak contributions in the atom-atom structure factors exactly cancel each other in the total scattering intensity, thus suppressing the pre-peak in agreement with the experimental findings. This cancellation is explained by drawing an analogy between the charge order found in ionic fluids and the segregated domain order. This finding opens new interpretation of the well known scattering prepeak observed in micro-emulsions. In particular, it implies that scattering experiment cannot detect homogeneous domain segregation, hence cannot lead to a proper microscopic description of atom-atom correlations in domain ordered mixtures.
\end{abstract}

\section{Introduction}

Aqueous mixtures of small quantities of tbutanol or n-alkyl polyglycol ether $\left(C_{n} E_{m}\right)$ show aggregation of these solute molecules in both cases [1, 2, 3, 4, 5, 6]. However, there is a considerable physico-chemical difference between these two types of aggregation. While tbutanol molecules form small aggregates about $1 \mathrm{~nm}$ wide 3], $C_{n} E_{m}$ molecules self-assemble into shapes called micelles about 5$10 \mathrm{~nm}$ wide[5]. The latter type of mixtures are called emulsions, while the former 
is a solution. In both cases, it is the dual amphiphilic/hydrophobic nature of the two types of molecules that produces the aggregate formation[7]. The microscopic structural differences between these two mixtures can be probed by radiation scattering [8, 9], such as light, Xray or small angle neutron scattering. The intensity $I(k)$ scattered off the micro-emulsion will show a Teubner-Strey behaviour [10] with an important pre-peak for wave vector $k_{P}$ in the range $k_{P} \approx$ $0.1-0.2 \AA^{-1}$, while for aqueous tbutanol mixture $I(k)$ will show a typical Ornstein-Zernike like behaviour, with no such pre-peak. The existence of the pre-peak is only weakly dependent of the nature of the scattering radiation type (light, neutrons, Xray), and seems to depend more on the type of aggregates 8 , 9]. The scattering data in both systems seems to suggest that the existence of a pre-peak could be related to the size of the aggregates.

Since a decade, however, a new aspect of the solution type mixtures has become apparent: the atom-atom pair correlation functions, as obtained in computer simulations, exhibit long range oscillations, which come from the existence of correlations between aggregated domains[11. Consequently, the corresponding atom-atom structure factors exhibit a domain pre-peak. This finding poses a problem: why this pre-peak does not contribute to the radiation scattered intensity $I(k)$ ?

One of the possible answers is that computer simulations could produce artifacts in the long range correlations, due to approximate molecular model, or statistical problems. However, this would be in variance with the fact that, for many neat liquids, computer simulations are able to predict scattering pre-peak in excellent agreement with experiments, for example neat alcohols [12] and neat room temperature ionic liquids [13, 14]. The origin of these pre-peaks has been related [13, 14, 15, 16] to the segregation of the charged (hydroxyl or ionic) and neutral (methyl or methylene) molecular groups. Furthermore, in such systems, the contribution of pre-peaks of the atom-atom structure factors to the total scattering pre-peak has been demonstrated. There is however an important difference between these neat systems and the mixtures mentioned above. As I have recently shown[17, the atom-atoms correlations of these neat systems do not show any long range domain oscillations corresponding to the segregation of the charged and neutral groups. Hence, the pre-peaks in such systems do not arise from segregated domain correlations, but correspond to the alternate disposition of the plus and minus charges within the charged group domain. This observation shows the principal difference between those neat systems which show scattering pre-peak and the case of mixtures presented here. Indeed, in the present case, both segregated species have atomic charges, such that, contrary to neat alcohols and ionic liquids, one cannot speak of charged group versus uncharged group segregation. What is even more problematic in the case of aqueous mixtures is precisely the absence of pre-peak in scattering experiments, versus their existence in atom-atom structure factors obtained from computer simulations.

The answer we provide here clarifies the origin of this discrepancy. We show that these long range domain oscillations in the atom-atom correlations are genuine physical features, but their contributions to the radiation scattering in- 
tensity vanish, because the various atom-atom contributions cancel each other. This cancellation is similar to that found in simple ionic liquids, where the charge order imposes out-of-phase long range oscillations [17, 18], hence drawing an analogy between charge order and domain order. In addition, the same analogy equally explains the existence of a pre-peak in the scattering in micellar systems, through a mechanism similar that produces a scattering prepeak in room-temperature ionic liquids, as compared to its absence in ordinary ionic liquids: the perturbation of the charge order by the uncharged methyl groups [17, 18. The equivalent mechanism in the case of domain order would be the perturbation of this order by the large interface between the two types of components. This new explanation gives a better microscopic foundation to the previous argument based on size antagonism between types of aggregates.

In the presentation below, we first recall the important details of the charge ordering process in different types of ionic liquids. Then, we present the case of aqueous-1pronanol as a prototype to show the nature of the domain order and the consequences on both the various atom-atom pair correlation function and the total scattering function. In the final part, we examine the consequences of the domain and charge order analogy for the understanding of the liquid-like order in complex liquids.

\section{Charge ordering}

Charge ordering is a crucial concept for this paper. Since it has been covered in a previous publications [17, 18, we will be brief about it in the presentation below. Charge ordering describes the very special form of order in simple ionic liquids, such as high temperature molten salts, for example. This special form of order is apparent from the correlation functions between like and unlike charge atoms, namely $g_{++}(r), g_{--}(r)$ and $g_{+-}(r)$, which are function of the atomatom separation distance $r$. Fig.1 shows a typical example taken from a model simulation of a ionic liquid, made of soft spheres of same diameter, which bear the charges of valence $z_{+}=+1$ and $z_{-}=-1$. In Fig.1a, it is seen that, past the details of the first neighbour correlations, the remainder of the correlations are exactly out of phase. This property translates into the following equality, which holds for large distances only, $r>r_{C} \approx 3.6 \AA$ :

$$
h_{++}=h_{--}=-h_{+-}
$$

where $h_{i j}=g_{i j}-1$. These equalities can be summarised in a unique one as

$$
\sum_{i j} h_{i j}=0 \quad r>r_{C}
$$

Correlation functions for uncharged atoms never obey this property, and are usually more of less in phase at large distances. Charge order is therefore a remarkable form or order in a disordered liquid. The origin of this order is naturally coming from the fact that like charges repel each other, while unlike 
charges attract each other, and in a disordered liquid, these local constraint leads to this special form of order. Charge order can equally be defined through the atom-atom structure factors, which are related to the Fourier transform of the correlation functions [19:

$$
S_{i j}(k)=\delta_{i j}+\rho \sqrt{x_{i} x_{j}} \int d \mathbf{r} h_{i j}(r) \exp (i \mathbf{k} \cdot \mathbf{r})
$$

where $x_{i}$ is the mole fraction of species $i$, and $\rho=N / V$ is the number density defined as the total number of atoms $N$ in the volume $V$. The functions $S_{++}(k)$, $S_{--}(k)$ and $S_{+-}(k)$ are shown in the inset of Fig.1b. Charge order is visible through the exact opposition of the peaks (shown by the red arrow), at the kvector $k \approx 1.67 \AA^{-1}$, which corresponds to the period of the long range oscillation in the $g_{i j}(r)$. The fact that the charge order peaks are exactly opposite in sign comes naturally from the equalities in Eq.(1).

The key information from charger order, which will be very helpful to understand domain order, is the exact cancellation of the structure factors charge order peaks. This is highlighted through the Bhatia-Thornton transformation[20], which holds only for binary mixtures. It consists in defining 2 new microscopic densities, the total local density $\rho_{N}(\mathbf{r})=\rho_{+}(\mathbf{r})+\rho(\mathbf{r})$ and the charge density $\rho_{Z}(\mathbf{r})=\left[z_{+} \rho_{+}(\mathbf{r})+z_{-} \rho_{-}(\mathbf{r})\right] / 2$, and introducing corresponding new structure factors $S_{A B}(k)=<\tilde{\rho}_{A}(k) \tilde{\rho}_{B}(-k)>$ through ensemble averages of the correlations of their Fourier transforms. In particular, one has for the density-density structure factor

$$
S_{N N}=\frac{1}{2}\left[S_{++}+S_{--}+2 S_{+-}\right]
$$

This structure factor $S_{N N}$ is equally represented (in orange) both in the main panel (c) and in the inset of Fig.1. The capital information from the main panel (c) is that the opposing peaks in the $S_{i j}$ do not appear in $S_{N N}$, due to their exact cancellation in the expression Eq, Conversely, these peaks appear in the charge-charge structure factor shown in the inset of panel (c). This cancellation crucially indicates that the density-density structure factor, which is actually the observable, does not contain the information about charge order. Hence, this structure factor looks like that of a random mixture, totally conceiling the fact that there is an underlying charge ordering beneath. In the next section, we will invoke a similar analogy for domain ordering, which will explain why domain order pre-peaks cancel in a similar way, which is the main message of this paper.

It is important to note that charge order is different from the global electroneutrality, although both are obviously related through the Coulomb interaction. Global electroneutrality is contained in the small-k limit of the structure factors, through the well-known Stillinger-Lovett sum rules [21]. So they concern $k=0$ behaviour of the structure factor. In contrast, charge order concerns the local distribution, as witness by both the medium-to-long range oscillations and the $k \neq 0$ wave vector where it manifests itself, and it may not necessarily obey electroneutrality, which is a global $(k=0)$ constraint. 
The various features of the charge ordering process shown here, are now used to demonstrate how domain-ordering follows a similar pattern to charge ordering.

\section{Domain ordering in aqueous 1propanol mix- tures}

We have studied by computer simulations the aqueous 1propanol mixtures, and in particular various atom-atom correlation functions and corresponding structure factors. This type of mixture corresponds to what we have named molecular emulsions [11], which show strong micro-heterogeneity, with water and solute segregated domains 22, 23. SPC/E water model 24 and TraPPe 1propanol model[25] were chosen. The focus is the $30 \% 1$ propanol aqueous mixture, since it is close to the maximum of the experimental Kirkwood-Buff integrals [26, 27, where maximum segregation effects are expected. The structure of this mixture has been previously studied by Xray and small angle neutron scattering experiments [28, 29, 30, 31] as well as computer simulations [32, 33], and both approaches revealed the clustering properties of these mixtures. It is important to note that none of these works have reported the existence of scattering or atom-atom domain correlation pre-peaks. The present simulations have been conducted in the isobaric ensemble by using the gromacs package 34. The temperature was maintained at $300 \mathrm{~K}$ through a Nosé-Hoover thermostat, and the pressure was let at 1atm using the Parrinello-Rahman barostat, with time constant 1ps. Various system sizes were investigated (see below). In each case, the system was equilibrated for $5 \mathrm{~ns}$, and production runs for $10 \mathrm{~ns}$. In order to properly sample long range oscillations due correlations between segregated domains, we have studied a system of $N=128000$ molecules, which corresponds to a box size of $L=195 \AA$. This is an unusually large number, but it is required, since lower system sizes do not allow a proper sampling of these domain-domain correlations -as shown further below.

Fig.2a shows all the atom-atom correlation functions $g_{a b}(r)$ (where $a$ and $b$ stands for the various atoms) for the aqueous mixture with $30 \% 1$ propanol. These are the 3 functions $g_{O_{W} O_{W}}, g_{O_{W} H_{W}}$ and $g_{H_{w} H_{w}}$ shown in blue for water (where the index $O_{W}$ and $H_{W}$ designate the water oxygen and hydrogen atoms, respectively), the 15 functions $g_{O O}, g_{O H}, g_{O M_{1}}, g_{O M_{2}}, g_{O M_{3}}, g_{O O}, g_{O H}$, $g_{O M_{1}}, g_{O M_{2}}, g_{O M_{3}}, g_{M_{1} M_{1}}, g_{M_{1} M_{2}}, g_{M_{1} M_{3}}, g_{M_{2} M_{2}}, g_{M_{2} M_{2}}, g_{M_{2} M_{3}}$ and $g_{M_{3} M_{3}}$ shown in green or 1propanol (where the index $O, H, M_{1}, M_{2}$ and $M_{3}$ designate the 1propanol oxygen, hydrogen and methylene/meythyl atoms, respectively), as well as the 15 cross correlation functions (shown in magenta) between these atoms, for a total of 33 functions.

The short and long range parts of the functions are displayed in separate vertical scales, in order to emphasize the different types of oscillations: atom-atom correlations at short range and domain-domain correlations at long range. In Fig.2a, the short range part (left panel) shows all the distinct features 
due to specificities of the various atoms. However, at long range(right panel), $r>r_{C} \approx 25 \AA$, all the specificity of the atomic features disappear and merge into 3 distinct features which depend only in the species-species correlations, corresponding respectively to the water-water, 1propanol-1propanol and water1 propanol correlations. It is clearly seen that these 3 type of species-species correlations show out-of-phase correlations between the like and cross correlations. The long range part of Fig.2a bears a striking resemblance with charge-ordering displayed in Fig.1, and we will consider here that these out of phase oscillations represent a "domain ordering", which is due to the micro-segregation of water and 1propanol(see snapshots in Fig.3).

Fig.2b shows the structure factors $S_{a b}(k)$ corresponding to the correlation functions $g_{a b}(r)$ shown in Fig.2a. The inset shows the domain pre-peak region enlarged. The pure 1propanol pre-peak is indicated by a red arrow, and is seen to occur at the k-vector larger than the domain pre-peak. Once again, we see that all the differences in the atomic details shows up essentially for $k>k_{C}=$ $2 \pi / r_{C} \approx 3.6 \AA^{-1}$, but for smaller k-values $k<k_{C}$, only the species-species specificity emerge into pre-peaks with opposing signs.. This finding proves that the domain-domain correlations are independent of the atomic details of the various molecular constituents. We have previously discussed such pre-peaks in the context of many other types of aqueous mixtures, and also non-aqueous mixtures 11]. But it is the first time that we relate such pre-peak to charge order, as seen in Fig.1, which they are clearly reminiscent of.

The micro-segregation of this system is illustrated in Fig.3, through snapshots for the $30 \%$ mixture of various sizes $N=2000, N=16000$ and $N=$ 128000 (each system is exactly the double of the size of the previous one). In all these three cases, the local segregation of domains is quite obvious, and these domains show an alternated dispositions, which strikingly resembles that of the charge ordering in the ionic liquid of the previous section, as shown in Fig.1a. There are important differences though. While the charges in the ionic liquid are localised within the atoms, the water and 1propanol domains do have have such sharp localisation. This feature has important heuristic implications that we will discuss later in Section 5. Although this "domain order" is much more loose than the strict charge order of the ionic liquid, the long range correlations hold an appealing analogy through the fact they appear to obey out-of-phase behaviour reminiscent of that captured through Eqs. (12) for the case of ionic liquids. This type of equality can be illustrated further through the analysis of the atom-atom structure factors $S_{a b}(k)$, shown in Fig.2b.

The domain order displayed in Figs.2 has also a striking similarity with the so-called bi-continuous and plumber phases found in micro-emulsions [35], which exhibit segregation of molecular species at a larger scales, but which occurs more often in presence of an water-oil-surfactant context. The important difference that these have with the actual system is the presence of better defined interface area, separating water-rich and oil-rich domains, and which is saturated with the surfactant [35. We conjecture that it is this segregation of a component into a lower dimensional area, which is responsible to the scattering Teubner Strey pre-peak[10] observed in such systems. We revisit this argument below in 
Section 5 .

The system size dependence is further illustrated in Fig.4a-b, where we show the 3 oxygen atom correlation functions $g_{O_{W} O_{W}}, g_{O O}$ and $g_{O_{W} O}$ (Fig.4a), as well as the corresponding structure factors (Fig.4b), calculated for the different system sizes shown in Fig.3. In both Fig.4a and Fig.4b, the insets focus on the respective domain-domain contributions. These figures show that the system size does not matter so much for the short range features. However, both insets show the dramatic differences coming from the long range part, which is sensitive to correct description of domain-domain correlations. For example, system size $N=2000$ leads to incorrect and too large $k=0$ predictions of the structure factors. This well known problem has been previously reported by us 22, 23. The $N=16000$ system seems appropriate since it gives results nearly similar to the $N=128000$ system, although the r-range does not extend beyond 48AA.

\section{Absence of radiation scattering pre-peak in domain- ordered systems}

\subsection{Expression for the scattered intensity}

One of the problem of predicting domain pre-peaks in the atom-atom structure factors is to explain why such pre-peaks are not experimentally observed in radiation scattering experiments[11]. We provide the answer here. The radiation scattering intensity $I(k)$ is formally defined through the Debye formula 36]

$$
I(k)=<\sum_{i, j} f_{i}(k) f_{j}(k) \exp \left(i \mathbf{k} \cdot\left(\mathbf{r}_{i}-\mathbf{r}_{j}\right)\right)>
$$

where the sum runs over all pairs of scattering atoms $i, j$, which are at respective spatial positions $\mathbf{r}_{i}$ and $\mathbf{r}_{j}$, the functions $f_{i}(k)$ are the atomic form factor for atom $i$ and depend on the type of radiation which is scattered, and the symbol $\langle\ldots\rangle$ designates an average over all possible positions of these atoms, which corresponds to a thermal average, or an ensemble average for calculational purposes. In practice, it is convenient to rewrite this expression in terms of the molecular species which contains the atoms 37. For a binary mixture, we introduce symbols $i, j$ to designate the molecular species index, and $a_{i}, b_{j}$ to designate the atoms of types $a$ and $b$ in respective molecules. Using the definition of the atom-atom structure factor :

$$
\rho \sqrt{x_{i} x_{j}} S_{a_{i} b_{j}}^{(M)}(k)=<\sum_{m_{a_{i} m_{b_{j}}}} \exp \left(i \mathbf{k} .\left(\mathbf{r}_{m_{a_{i}}}-\mathbf{r}_{m_{b_{j}}}\right)\right)>
$$

where the sum runs over all atoms of type $a_{i}, b_{j}$, and $x_{i}=N_{i} / N$ is the mole fraction of molecular species $i$. In the equation above, the atom-atom structure factor $S_{a b}^{(M)}(k)$ is the not the same as that which appears in Eq. 3 , since it contains contributions from the intra-molecular contributions as well, hence the superscript (M) for molecular. Indeed, the sum in Eq.(6) contains also atom 
pairs in the same molecule. It can be shown, in case of atoms rigidly bound inside a molecule, that this contribution in Eq. (6) comes down to the Bessel function $j_{0}\left(k d_{a b}\right)=\sin \left(k d_{a b}\right) / k d_{a b}$, where $d_{a b}=\left|\mathbf{r}_{a}-\mathbf{r}_{b}\right|$. This function is the same as the $\mathrm{W}$-matrix, with elements $w_{a b}(k)=j_{0}\left(k d_{a b}\right)$, which appears in the Site-Site Ornstein-Zernike theory [19], and which contain the intra-molecular contribution to the pair correlation function. The link with the structure factor defined through Eq. 3. 3 and the atom-atom pair correlation function $g_{a_{i} b_{j}}(r)$ is then

$$
S_{a_{i} b_{j}}^{(M)}(k)=w_{a_{i} b_{j}}(k)+\rho \sqrt{x_{i} x_{j}} \int d \mathbf{r}\left[g_{a_{i} b_{j}}(r)-1\right] \exp (i \mathbf{k} \cdot \mathbf{r})
$$

which represents a generalisation of Eq. (3) to molecular systems. By noting that the form factors $f_{i}(k)$ do not depend on the thermal average $<$. $>$, we can rewrite the Debye formula into:

$$
I(k)=\rho \sum_{i j} \sqrt{x_{i} x_{j}} \sum_{a_{i} b_{j}} f_{a_{i}}(k) f_{b_{j}}(k) S_{a_{i} b_{j}}^{(M)}(k)
$$

which is convenient to recalculate the scattered intensity from the atom-atom pair correlation functions and the corresponding atom-atom structure factors. It is interesting to note that this expression is similar to the Pings-Waser (PW) formula 37] generically used by many authors, but which does not contain the intra-molecular part $w_{a b}(k)$. The present derivation shows both the origin of this term and how to incorporate this contribution into the PW expression through the correct expression Eq. (7). The strict PW formula is recovered by setting $W_{a b}(k)=\delta_{a b}$. The expression in Eq. (8) applies both for Xray and neutron scattering, when appropriate form factors are used. In the case of neutron scattering, $I(k)$ represents only the incoherent part of the scattering.

\subsection{Application to the aqueous-1propanol mixtures}

We now compute the Xray scattering intensity from various atom-atom pair correlation functions and structure factors shown in Fig.2. The form factors are taken from the scattering data 38 . Fig. 5 shows the total $I(k)$, as well as the 3 species-species contributions, namely water-water (blue), propanol-propanol (green) and water-propanol (magenta) contributions to $I(k)$. The dashed red line represent the negative of the sum of water-water and propanol-propanol contributions, which should match the magenta curve if exact cancellation should occur, which is seen to be the case in the pre-peak region. From the main panel of Fig.5, it is clearly seen that each of these contributions in the pre-peak region are 2 orders of magnitude larger than the total $I(k)$. However, the total contribution totally cancels the pre-peak, as can be seen in the expanded view of $I(k)$ reported in the top inset. This inset shows that only the 1 propanol and water main peak are dominant, at $k \approx 0.65 \AA^{-1}$ and $k \approx 1.45 \AA^{-1}$, respectively. This cancellation is a striking result for several reasons. First of all, it is consistent with the known absence of pre-peak in the experimental Xray scattering data 
for this particular system Ref.[31]. Secondly, in order for the cancellation of such huge pre-peak contribution to happen, despite the fact that experimental form factors are used in conjunction with calculated structure factors, there must be a fine tuned adjustment of these cancellations. This fact proves that domain order is a very robust physical phenomena.

Then, if one considers the relative good agreement between the calculated $I(k)$ and the experimental one, as shown in the lower inset of Fig.5, and considering the fact that the form factors are taken from the experimental data, this agreement enforces the hypothesis that the simulated atom-atom structure factors must be close to the experimental data - if such data could be measured. Indirectly, it confirms that the classical model representation must be good enough to provide the observed cancellation. Finally, it is important to realise that the absence of the pre-peak in the experimental data does not allow to infer the existence of domain correlations in each of the partial contributions, since these cannot be observed individually. This is a dramatic finding, since it shows that the existence of the domain-domain correlations can only be predicted from theory and apparently against experimental observation.

The lower inset of Fig.5 shows a comparison between the calculated $\Delta I(k)$ and the Xray data from Ref.[31] (shown in blue). It is seen that the agreement is quite fair, including in the pre-peak region. The agreement is better on the various peak positions than in the data itself, which implies that the size of the molecules are well described by the models but their distribution is slightly dephased with respect to real one. The data reported in Ref.31 is $\Delta I(k)=$ $I(k) / I_{I d}(k)-1$, where $I_{i d}(k)$ corresponds to the ideal part of the expression in Eq. (8), in other words when the structure factors $S_{a b}(k)$ in Eq. (7) are replaced by the first term in this equation, namely $w_{a b}(k)$.

We have equally computed the neutron scattered intensity by using the appropriate form factors for deuterated water and 1propanol, namely $\mathrm{D}_{2} \mathrm{O}$ and $O D C_{3} H_{7}$, and the cancellation of the domain pre-peaks occurs once again. The total $\mathrm{I}(\mathrm{k})$ looks very much like that reported in the upper inset of Fig.5.

It is interesting to see how domain order affect the scattered intensity. For this, we select in Eq. (8) the k-vectors under the pre-peak contributions, where only the species-species contributions are seen and all atom-atom details are washed out. For this range of k-vectors, the various atom-atom structure factors of a given species pair are strictly similar:

$$
S_{a_{i} b_{j}}(k)=S_{i j}(k) \text { for } 0<k<k_{D}
$$

where $k_{C}$ is the maximum k-vector for which the domain order pre-peak is numerically distinctively defined. For this k-vector range, the Debye expression in Eq. 8) becomes

$$
I(k)=\rho \sum_{i j} \sqrt{x_{i} x_{j}} F_{i}(k) F_{j}(k) S_{i j}(k) \text { for } 0<k<k_{C}
$$

where the effective form factor functions $F_{i}(k)$, which depend now on species, rather than atoms are defined as: 


$$
F_{i}(k)=\sum_{a_{i}} f_{a_{i}}(k)
$$

This expression is very similar to the form factor one would get in case of approximating a super-atom made of all the atoms inside a single molecule CITE. This type of transformation is used to describe the methyl and methylene group as super atoms, where the form factor of the united atom is approximated as $f_{M}=f_{C}+n f_{H}$, and $f_{C}$ and $f_{H}$ are the form factors of the carbon and hydrogen atoms, respectively, with $n=2,3$ for the methylene and methyl pseudo atoms, respectively.

What Eq. (10) tells us is that pre-peak k-vector region is dominated by scattering of the molecular species as pseudo-atoms, indifferently to the atomic details. In that, it is strictly similar to the ionic liquid model, with only 2 mono-atomic species, with the appropriate pseudo-atom form factor $F_{i}(k)$. It is interesting to see that, for a binary component, the expression in Eq. 10 is similar to the Bathia-Thornton structure factor $S_{N N}$ in Eq.(4), with $I(k)=$ $\rho\left[F_{1}^{2} S_{11}+F_{2}^{2} S_{22}+2 F_{1} F_{2} S_{12}\right]$, for $0<k<k_{D}$.

\section{Discussion}

Perhaps the most important consequence of domain order is the inability of predicting this type of order from experimental scattering methodology, as confirmed by the various experimental findings of the aqueous-1propanol mixtures 29 , 31. Indeed, if the experimental $I(k)$ should be used to obtain the individual atom-atom structure factors and correlation functions, one would not be able to find the large pre-peaks which correspond to the domain-domain correlations, since this information is absent in $I(k)$. Since scattering data for $I(k)$ exist for several system of aqueous mixtures and other such system exhibiting microheterogeneity, the tentative to obtain correlation functions and compare them with simulation data are most probably erroneous, at least in what concern the long range part. This deficiency is more a fundamental problem than a technical one. Indeed, it means that one has to infer the existence and the microscopic details of molecular segregation solely from models and theory. Therefore, one should seek a better theoretical description of domain segregation, as well as its consequences.

Micro-segregation is analogous to charge order, in that the segregated domains are disposed in quasi alternate fashion -a checker board type order, in order to maximize the segregation, without leading to full phase separation. However, charge order concerns particle with fixed shapes, and occurs at the level of the particles themselves. In contrast, domain order concerns fuzzy molecular domains, with a certain degree of cross mixing which depends on the nature of the interactions. This is the reason why domain order is only observed in the long range part of the pair correlation functions. One can imagine domain order as being a smooth distortion of charged particles into fuzzy domain, hence going from a particle representation into a field representation. Strictly speaking, 
domain order is the field representation of charge order.

There is an important difference concerning the valence of the particle charges, which dictates charge order, and the domain valence, which dictates domain ordering. Indeed, like-charges repel each other while unlike-charges attract each other, leading to a large peak in the cross correlation function. Conversely, in domain ordering, it is the like correlations which exhibit the high peak at atomic contact, while cross correlations are depressed at contact. In terms of interactions, if a strict mapping with the Coulomb interactions should be made, this behaviour would correspond to pure imaginary charges. If a ionic liquid with imaginary charges would be used, it will lead to immediate demixing of each valence. Therefore, one requires a supplementary mechanism to maintain the particles into charge order. This mechanism is provided here through the hydrophobic-hydrophilic interaction balances, which maintain the domain order. Moreover, domain ordering is seen as a part of the atom-atom correlations, namely the long range part. In order to describe this contribution, one could resort to a field theory description, by assigning a phase to each of the fields corresponding to atom-atom distribution functions.

It is interesting that the demonstration of domain order requires extensive computer simulations, nearly at the edge of what can be done in desktop PCtype workstations. Moreover, it seems reasonable to think that most of softmatter system experience such domain order at various degrees of extent. In view of the computer power required in the present case of aqueous-1propanol mixture, a system which cannot be considered as particularly challenging, one wonders at the type of resources that could be required to account for domain order in simulation of realistic systems as those found in soft-matter. Conversely, one wonders how much importance domain order can have in such system, and to what extent it can be neglected. These are subjects for subsequent investigations, which are newly opened by the present investigation.

When going from simple ionic liquids, such as molten $\mathrm{NaCl}$, to room temperature ionic liquids, such as ethylammonium-nitrate, for example, the particles change from simple charged atoms to complex molecules, which contain uncharged methyl and methylene groups [39, 40]. These groups perturb the global homogeneity of the charge ordering found in simple ionic liquids, and the loss of global homogeneity gives rise to a pre-peak both in the atom-atom structure factors and the total scattered intensity[17, 18, 39, 40]. Similarly, when going from molecular emulsions to micro-emulsions, we conjecture that the global domain order is perturbed by the change in the nature of the aggregates. Direct micelles contain extended water impregnated outer interface, sharply separated from the inner oily core [4]. Inverse micelles also have well separated polar inner cores from the fuzzy and hairy outer core made of the hydrophobic tails [7]. This is a sharp change in the nature of the type of aggregates found in molecular emulsions. We conjecture here that is this change which produces a non canceling pre-peak in the total scattered intensity. Demonstrating this conjecture would require computer simulations beyond desktop capabilities. Yet, many systems found in soft-matter or biomaterial context, contain structural aggregates similar to micelles [41, 42, 43]. The fact that scattering experiments 
in such systems are able to predict scattering pre-peak[43] does not necessarily imply that they can provide a better microscopic description. Indeed, there are probably hidden cancellation mechanisms beneath the apparent pre-peak of micro-emulsions, which would require further investigations similar to that conducted herein. The microscopic relation between micelle structure and scattering data remains to be re-investigated in the light of the present finding. We conjecture that the present theoretical descriptions of micelle formation, which never take into account domain ordering, are analogous to the Debye-Huckel description of ionic liquids, which does not account for charge order, but describes correctly the screening of charges. A new theory of micro-emulsions, which accounts for domain order, is yet to be developed.

Another point concerns the actual physical impact of domain order in terms of radiation scattering for the molecules themselves. In other words, how does the fact that radiation scattering does not witness a particular spatial frequency, despite the existence of domain segregation, influences the actual physico-chemical properties of the molecules themselves, specially if some of them are radiation sensitive? The answer to this question could have a interesting consequences in the bio-context, for example. The same question can be asked at the more general point of view of any form of micro-segregated matter.

\section{Conclusion}

The most important conclusion of this work is the fact that micro-segregation in molecular emulsions induces domain order, which is a form of macroscopic homogeneity, rescaled at the level of the segregated domains as pseudo-molecular grains. It is this rescaled homogeneity which is the principal reason why scattering experiments cannot detect the underlying domain segregation. This apparent homogeneity is similar to that found in atomic ionic liquid, where the particles do not experience the random disordered distribution seen in simple binary mixtures, but the charge order dictated by the Coulomb interactions. It is this form of order-within-disorder, which produces the apparent homogeneity of these systems. Micro-segregated mixtures have the same type of order, hence they look more homogeneous that they actually are, at least from the point of view of scattered radiations. This apparent homogeneity can be destroyed by super-structures such as micelles, which appear when going from molecular emulsions to micro-emulsions. This is not a simple change in the size of aggregates, as previously thought, but a topological change in the conformal structure of the aggregate, which in particular induces a change in the homogeneity of the system. Finally, we have emphasized that the data from scattering experiments cannot lead to a complete atom-atom microscopic structure of complex microheterogeneous liquid mixtures, in severe contrast with the computer simulation calculations. 


\section{References}

[1] T. M. Bender and R. Pecora, A Dynamlc Light Scattering Study of the tert -Butyl Alcohol-Water System, J. Phys. Chem. 90, 1700 (1986)

[2] J. L Finney, D. T Bowron and A. K Soper, The structure of aqueous solutions of tertiary butanol, J. Phys.:Cond. Mat. 12, A123 (2000)

[3] D. Subramanian and M. Anisimov, Resolving the Mystery of Aqueous Solutions of Tertiary Butyl Alcohol, J. Phys. Chem B 115, 9179 (2011)

[4] L. J. Magid in Nonionic Surfactants, ed. M. J. Schick and Marcel Dekker, inc. New York (1987)

[5] G. D'Arrigo, R. Giordano and J. Teixeira, Small-angle neutron scattering studies of aqueous solutions of short-chain amphiphiles, Eur. Phys. J. E 10, $135(2003)$

[6] R. R. Durand, S. M. Hajji, R. Coudert, A. Cao and E. Taillandier, Study of Micellar Solutions of Octanetriol-1,2,3 by Light Scattering Techniques , J. Phys. Chem. 92, 1222 (1988)

[7] C. Tanford, Science, 200, 1012 (1978)

[8] B. J. Berne and R. Pecora, Dynamic light scattering, Dover, New-York (1976)

[9] S.-H. Chen and P. Tartaglia, Scattering Methods in Complex Fluids, Cambridge Press (2015)

[10] M. Teubner and R. Strey, J. Chem. Phys. 87, 3195 (1987)

[11] A. Perera, From solutions to molecular emulsions, Pure and Appl. Chem. 88,189 (2016)

[12] M. Tomšič, A. Jamnik, G. Fritz-Popovsi, O. Glatter and L. Vlček, Structural Properties of Pure Simple Alcohols from Ethanol, Propanol, Butanol, Pentanol, to Hexanol: Comparing Monte Carlo Simulations with Experimental SAXS Data, J. Phys. Chem. B 111, 1738 (2007)

[13] C. S. Santos, H. V. R. Annapuredy, N. S. Murthy, H. K. Kashyap, E. W. Castner Jr. and C. J. Margulis, Temperature-dependent structure of methyltributylammonium bis(trifluoromethylsulfonyl)amide: $\mathrm{X}$ ray scattering and simulations, J. Chem. Phys. 134, 064501 (2011)

[14] M. Macchiagodena, F. Ramondo, A. Triolo, L. Gontrani and R. Caminiti, Liquid Structure of 1-Ethyl-3-methylimidazolium Alkyl Sulfates by X-ray Scattering and Molecular Dynamics , J. Phys. Chem. B 116, 13448 (2012)

[15] A. A. H. Pádua, M. F. Costa Goms and J. N. A. Canongia Lopes, Molecular Solutes in Ionic Liquids: A Structural Perspective, Acc. Chem. Res. 40, 1087 (2007) 
[16] Y. Wang, W. Jiang, T. Yan and G. A. Voth, Understanding Ionic Liquids through Atomistic and Coarse-Grained Molecular Dynamics Simulations , Acc. Chem. Res. 40, 1193 (2007)

[17] A. Perera, Charge ordering and scattering pre-peaks in ionic liquids and alcohols , Phys. Chem. Chem. Phys. 19, 1062 (2017)

[18] A. Perera and R. Mazighi, Simple and complex forms of disorder in ionic liquids , J. Mol. Liq. 210, 243 (2015)

[19] J. P. Hansen and I. McDonald, Theory of Simple Liquids, Academic, London (1986).

[20] A. B. Bhatia and D. E. Thornton, Structural Aspects of the Electrical Resistivity in Binary Aloys, Phy. Rev. B 2, 3004 (1970)

[21] F. H. Stillinger and R. Lovett, General Restriction on the Distribution of Ions in Electrolytes, J. Chem. Phys. 49, 1991 (1968)

[22] B. Kežić and A. Perera, Aqueous tert-butanol mixtures: A model for molecular-emulsions, J. Chem. Phys. 137, 014501 (2012)

[23] B. Kežić and A. Perera, Revisiting aqueous-acetone mixtures through the concept of molecular emulsions, J. Chem. Phys. 137, 134502 (2012)

[24] H. J. C. Berendsen, J. R. Grigera and T. P. Straatsma, The Missing Term in Effective Pair Potentials, J. Phys. Chem. 91, 6269 (1987)

[25] B. Chen, J. J. Potoff and J. I. Siepmann, Monte Carlo Calculations for Alcohols and Their Mixtures with Alkanes, J. Phys. Chem B 105, 3063 (2001)

[26] E. Matteoli and L. Lepori, Solute-solute interactions in water. II. An analysis through the Kirkwood-Buff integrals for 14 organic solutes, J. Chem. Phys. 80, 2856 (1984)

[27] A. Perera; F. Sokolic, L. Almasy and Y. Koga, Kirkwood-Buff integrals of aqueous alcohol binary mixtures, J. Chem. Phys. 124, 124515 (2006)

[28] G. H. Grossmann and K. H. Ebert, Formation of Clusters in 1PropanoVWater-Mixtures , Ber. Bunsenges. Phys. Chem. 85, 1026 (1981)

[29] H. Hayashi, K. Nishikawa and T. Iijima, Small-Angle X-ray Scattering Study of Fluctuations in 1-Propanol-Water and 2-Propanol-Water Systems, J. Phys. Chem. 94, 8334 (1990).

[30] M. Misawa, Mesoscale structure and fractal nature of 1-propanol aqueous solution: A reverse Monte Carlo analysis of small angle neutron scattering intensity , J. Chem. Phys. 116, 8463 (2002) 
[31] T. Takamuku, H. Maruyama, K. Watanabe and T. Yamaguchi, Structure of 1-Propanol-Water Mixtures Investigated by Large-Angle X-ray Scattering Technique, J. Sol. Chem. 33, 641 (2004)

[32] I. Akiyama, M. Ogawa, K. Takase, T. Takamuku, T. Yamaguchi and N. Ohtori1, Liquid Structure of 1-Propanol by Molecular Dynamics Simulations and X-Ray Scattering, J. Sol. Chem. 33 ,797 (2004)

[33] A. B. Roney, B. Space; E. W. Castner, L. Napoleon and P. B. Moore, A Molecular Dynamics Study of Aggregation Phenomena in Aqueous nPropanol , J. Phys. Chem. B 108, 7389 (2004)

[34] D. van der Spoel, E. Lindahl, B. Hess, G. Groenhof, A.E. Mark, H.J.C. Berendsen, GROMACS: Fast, Flexible, and Free , J. Comp. Chem. 26 (2005) 1701.

[35] U. S. Schwarz and G. Gompper, Bicontinuous surfaces in self-assembling amphiphilic systems, In: Morphology of Condensed Matter: Physics and Geometry of Spatially Complex Systems, Eds. K. R. Mecke and D. Stoyan, Springer Lecture Notes in Physics Vol. 600, 107-151 (2002)

[36] P. Debye, Zerstreuung von Rontgenstrahlen. Annalen der Physik 351, 80923 (1915)

[37] C. J. Pings and J. Waser, Analysis of Scattering Data for Mixtures of Amorphous Solids or Liquids , 48, 3016 (1968)

[38] International Tables for Crystallography, ed. E. Prince, Inter- national Union of Crystallography, 2006, vol. C.

[39] H. V. R. Annapureddy, H. K. Kashyap, P. M. De Biase, and C. J. Margulis, What is the Origin of the Prepeak in the X-ray Scattering of ImidazoliumBased Room-Temperature Ionic Liquids? J. Phys. Chem. B 114, 16838 (2010)

[40] H. K. Kashyap, J. J. Hettige, H. V. R. Annapureddy and Claudio J. Margulis, SAXS anti-peaks reveal the length-scales of dual positive-negative and polar-apolar ordering in room-temperature ionic liquids, Chem. Commun. 48, 5103 (2012)

[41] B. Jacrot, The study of biological structures by neutron scattering from solution, Rep. Prog. Phys. 39, 911 (1976)

[42] N. Allec, M. Choi N. Yesupriya, B. Szychowski, M. R. White, M. G. Kann, E. D. Garcin, M.-C. Daniel and A. Badano, Small-angle X-ray scattering method to characterize molecular interactions: Proof of concept , Nature, Scientific Reports 5, 12085, DOI: 10.1038/srep12085 (2015)

[43] L. Boldon, F. Laliberte and L. Liu, Review of the fundamental theories behind small angle X-ray scattering, molecular dynamics simulations, and relevant integrated application, Nano Reviews, 625661 (2015) 


\section{Figure captions}

Fig.1 (a) Snapshot of charge order for the ionic liquid characterized by the parameters below the picture. (b) Pair correlation functions and corresponding BT functions (inset; see text). (c) Structure factors and corresponding BT functions (inset; see text). The red arrows indicates the pre-peak position.

Fig.2 (a) Atom-atom pair correlation functions of the aqueous-1propanol $30 \%$ mixture. The functions are displayed into 2 different distance scale (as indicated by the blue arrows) separating the short and long range parts. the inset shows details of the correlations at atomic contact. Blue is for water-water correlations, magenta for propanolpropanol and green for cross correlations. The functions $g_{O_{w} O_{w}}(r)$, $g_{O_{P} O_{P}}(r)$ and $g_{O_{W} O_{P}}(r)$, corresponding to the correlations between the oxygen atoms of water and 1propanol, are highlighted in thicker lines. (b) Corresponding atom-atom structure factors with same line and color conventions. The red arrow indicates the position of the neat propanol pre-peak. The inset represent a zoom over the pre-peak part.

Fig.3 Snapshots of the systems for various sizes: (a) $\mathrm{N}=2000$, (b) $\mathrm{N}=16000$ and (c) $\mathrm{N}=128000$, showing the segregation of the polar $\mathrm{OH}$ groups and the oily methyl groups (in cyan). The water oxygen and hydrogen atoms are shown in red and white, respectively, those of 1propanol in blue and grey, respectively, and the methyl/methylene groups are shown in cyan.

Fig.4 System size dependence of the atom-atom pair correlation functions (a) and corresponding structure factors (b), illustrated for the three oxygen-oxygen correlations, namely water-water (WW), 1propanol1 propanol (PP) and cross (WP). The data for system size 128000 is shown in full lines, 16000 in dotted lines and 2000 in dashed lines. Corresponding WW correlations are colored in blue, cyan and purple, respectively, for PP correlations in green, yellow and grass, and cross WP correlations in magenta, red and brown, respectively. In (b), the structure factor of pure water is shown as thin black curve.

Fig.5 Xray scattering intensity for the $30 \%$ 1propanol in the aqueous mixture, as computed from collecting the atom-atom structure functions calculated in the simulations, through Eq.(8). The main panel shows the 3 partial species-species contributions to $\mathrm{I}(\mathrm{k})$ (blue for water, green for propanol and magenta for cross) and the calculated $I(k)$ in black (for the dotted red line, see text). The calculated $\mathrm{I}(\mathrm{k})$ is reproduced in magnified scale in the upper inset. The lower inset shows a comparison with experiments (shown in blue from Ref. 31]) in blue of the quantities $\Delta I(k)=k\left[I(k) / I_{I d}(k)-1\right]$ (see text). 
(a)

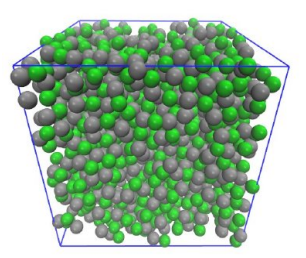

$\sigma_{1}=\sigma_{2}=3 \AA \quad \epsilon_{1}=\epsilon_{2}=80 k_{B}$

$\rho=0.9 \quad \mathrm{~T}=2000 \mathrm{~K}$ (b)

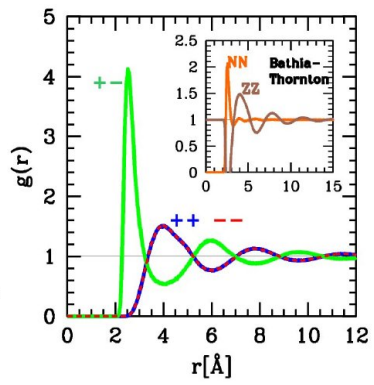

(c)

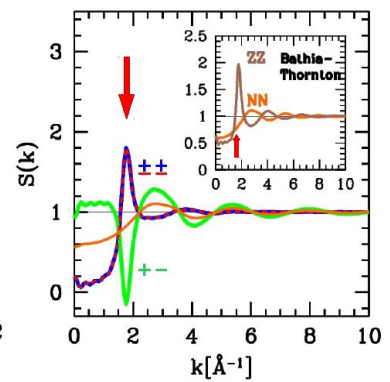

Fig.1. (a) snapshot of charge order for the ionic liquid characterized by the parameters below the picture. (b) Pair correlation functions and corresponding BT functions (inset; see text). (c) Structure factors and corresponding BT functions (inset; see text). The red arrows indicates the pre-peak position. 
(a)

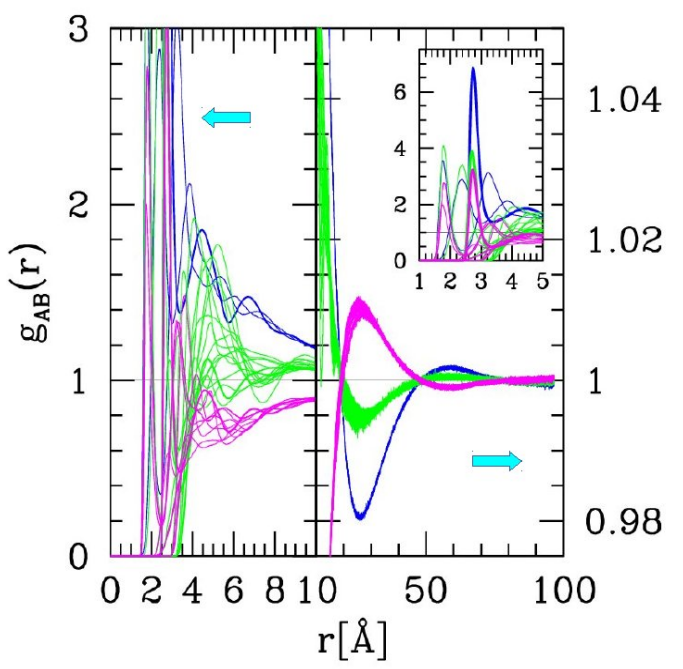

(b)

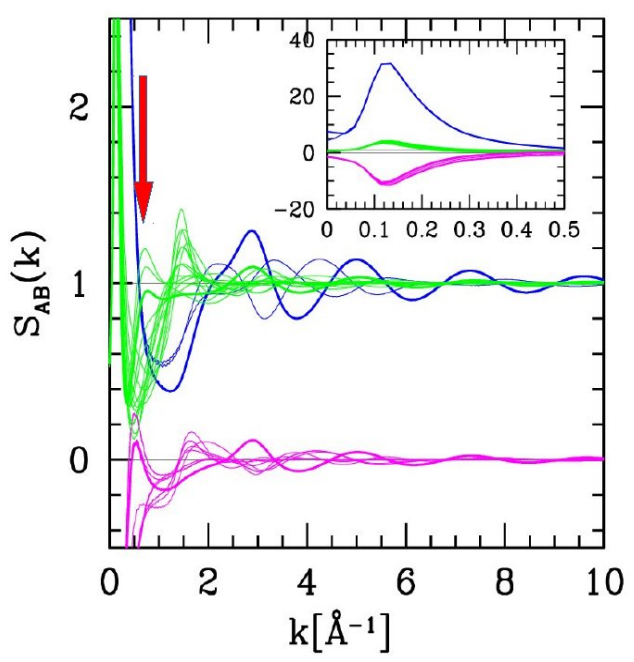

Fig.2. (a) Atom-atom pair correlation functions of the aqueous-1propanol $30 \%$ mixture. The functions are displayed into 2 different distance scale (as indicated by the arrows) separating the short and long range parts. the inset shows details of the correlations at atomic contact. Blue is for water-water correlations, green for propanol-propanol and magenta for cross correlations. The functions $g_{O_{w} O_{w}}(r), g_{O_{P} O_{P}}(r)$ and $g_{O_{W} O_{P}}(r)$, corresponding to the correlations between the oxygen atoms of water and 1propanol, are highlighted in thicker lines. (b) Corresponding atom-atom structure factors with same line and color conventions. The inset represent a zoom over the pre-peak part. 


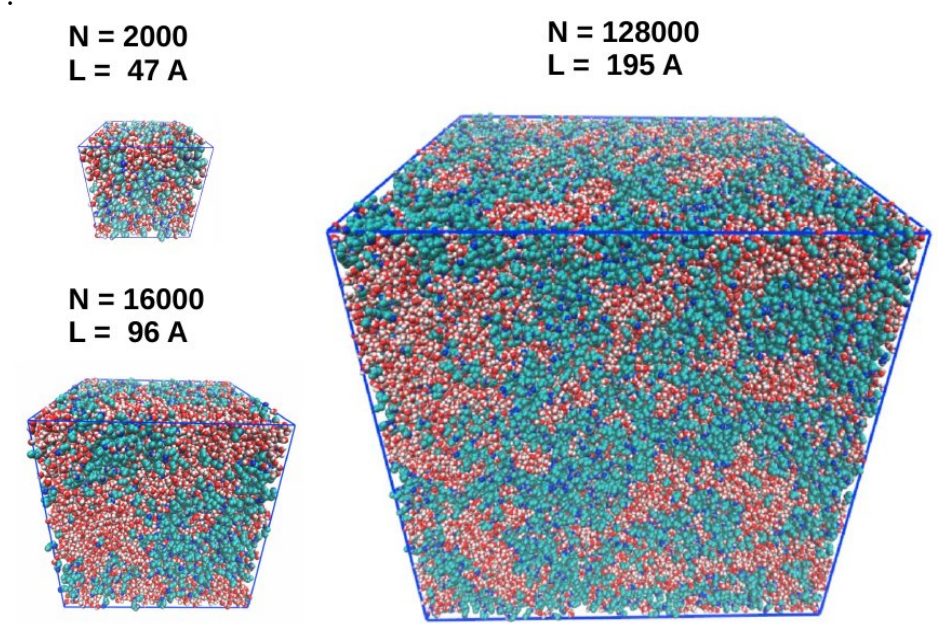

Fig.3. Snapshots of the systems for various sizes: (a) $\mathrm{N}=2000$, (b) $\mathrm{N}=16000$ and (c) $\mathrm{N}=128000$, shown the segregation of the polar $\mathrm{OH}$ groups and the oily methyl groups (in cyan). The water oxygen and hydrogen atoms are shown in red and white, respectively, those of 1propanol in blue and grey, respectively, and the methyl/methylene groups are shown in cyan. 

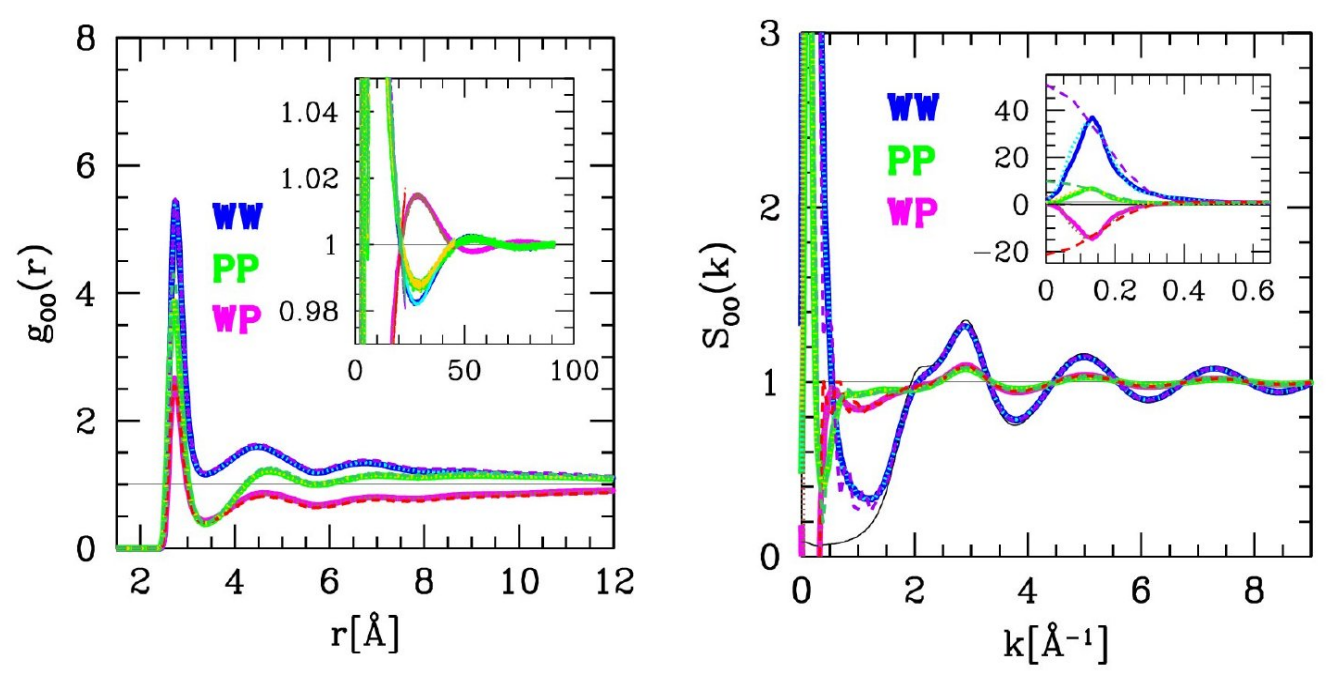

Fig.4. System size dependence of the atom-atom pair correlation functions (a) and corresponding structure factors (b), illustrated for the three oxygenoxygen correlations, namely water-water (WW), 1propanol-1propanol (PP) and cross (WP). The data for system size 128000 is shown in full lines, 16000 in dotted lines and 2000 in dashed lines. Corresponding WW correlations are colored in blue, cyan and purple, respectively, for PP correlations in green, yellow and grass, and cross WP correlations in magenta, red and brown, respectively. In (b), the structure factor of pure water is shown as thin black curve. 


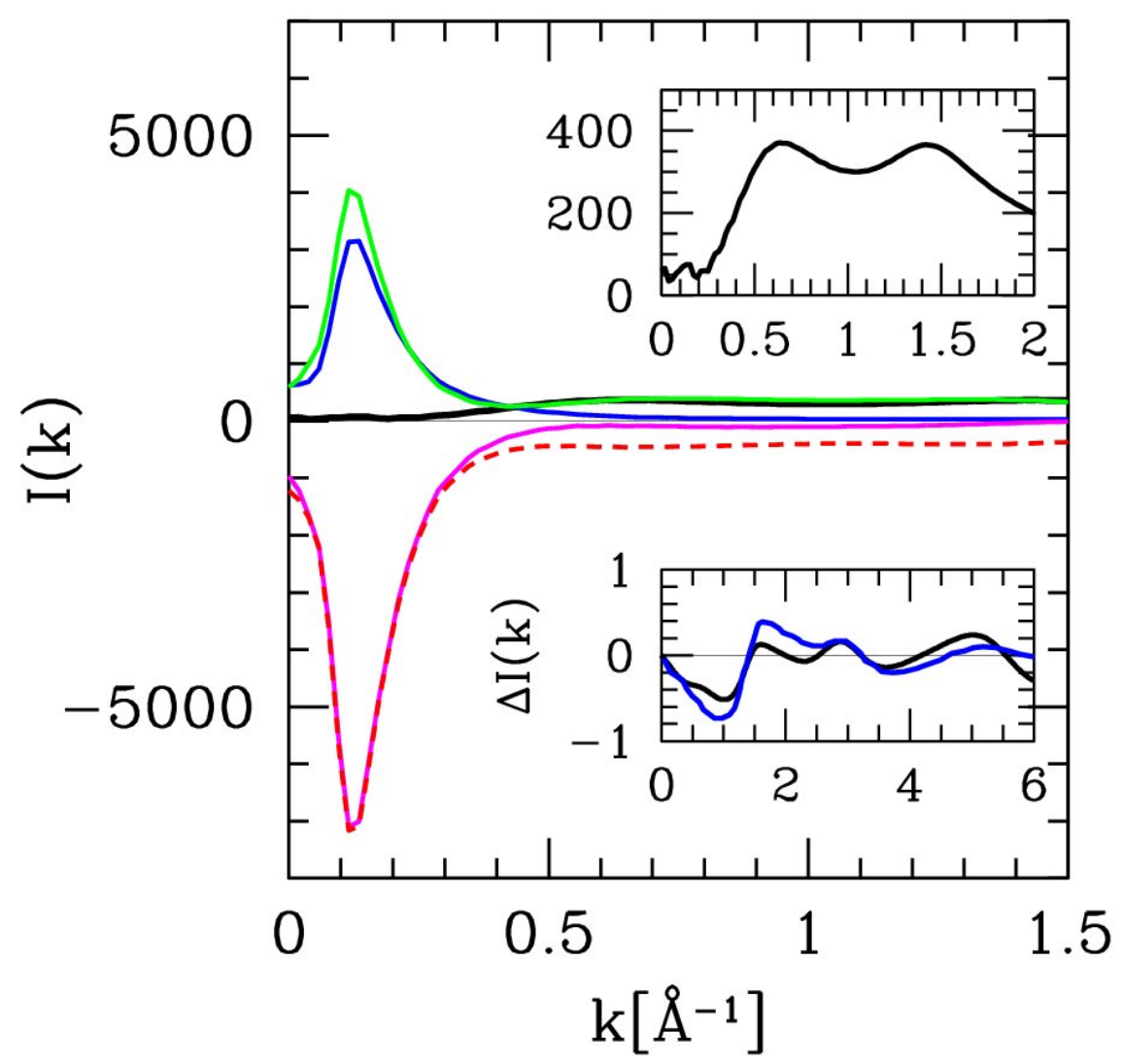

Fig.5. Xray scattering intensity for the $30 \%$ 1propanol in the aqueous mixture, as computed from collecting the atom-atom structure functions calculated in the simulations, through Eq.(8). The main panel shows the 3 partial speciesspecies contributions to $\mathrm{I}(\mathrm{k})$ (blue for water, green for propanol and magenta for cross) and the calculated $I(k)$ in black (for the dotted red line, see text).The calculated $\mathrm{I}(\mathrm{k})$ is reproduced in magnified scale in the upper inset. The lower inset shows a comparison with experiments (shown in blue from Ref. 31]) of the quantities $\Delta I(k)=k\left[I(k) / I_{I d}(k)-1\right]$ (see text). 